\title{
Atitude de consumidores brasileiros sobre o bem-estar animal
}

\section{Attitude of Brazilian consumers on animal welfare}

\author{
Bruna Maria Remonato Franco, Elaine Cristina de Oliveira Sans*, Maria Alice Schnaider, Vanessa Souza Soriano, \\ Carla Forte Maiolino Molento \\ Laboratório de Bem-estar Animal (LABEA), Universidade Federal do Paraná (UFPR), Curitiba, PR, Brasil
}

\section{Resumo}

Os objetivos deste trabalho foram estudar e identificar as atitudes dos consumidores de produtos de origem animal com ênfase no bem-estar animal (BEA), possíveis vantagens do consumo de alimentos oriundos de melhores práticas de bem-estar e fatores que possam influenciar as decisões de compra. Para isto, foi realizada uma pesquisa online com 402 consumidores de produtos de origem animal provenientes das cinco regiões brasileiras. Os respondentes afirmaram que o tema BEA recebe pouca importância no país, avaliando as condições dos animais de produção no Brasil como piores quando comparadas às de outras nações, e atribuíram aos produtores rurais a principal responsabilidade quanto ao assunto. Os respondentes demonstraram uma desassociação entre o alimento e o animal que o originou, porém reconheceram que há influência na promoção do BEA ao adquirirem produtos diferenciados para tal atributo. Do ponto de vista dos respondentes, a disponibilidade de produtos com maior grau de bem-estar, e as informações disponíveis na rotulagem quanto à forma com que os animais são criados, constituem gargalos para a aquisição de tais produtos. 0 preço pode ser considerado um fator importante, visto que a maioria dos respondentes aceitaria pagar $10 \%(32,3 \%)$ ou $25 \%(24,6 \%)$ a mais em produtos produzidos com maior grau de bem-estar. Com base nos resultados, os respondentes brasileiros se importam com o BEA, porém, diversos fatores podem ser entraves para que exerçam sua preferência ética no comportamento de compra, como falta de informação para o consumidor antes e no momento da compra, baixa disponibilidade dos produtos, desassociação do produto com o animal de origem e custo elevado.

Palavras-chave: Animais de produção. Demanda. Disponibilidade. Mercado consumidor. Produtos de origem animal.

\section{Abstract}

The objectives of this study were to study and to identify consumer attitudes regarding animal welfare, possible advantages of food consumption from better welfare practices, and factors that may influence purchasing decisions. For this purpose, an online research with 402 consumers from five Brazilian region was conducted. 
The respondents believed that the subject animal welfare receives scant attention in the country and estimated farm animal welfare in Brazil as worse than in other nations. Producers were considered responsible for farm animal welfare. The respondents revealed a disassociation between the food and the animal that originated it, however they perceived their influence on animal welfare when they purchase welfare-friendly products. From the respondents's point of view, the availability of welfarefriendly products and the information about how farm animals are raised on products labels are considered barriers for the purchase of these products. The price is also considered an important factor, since the majority of respondents would pay up to $10 \%$ (32.3\%) or $25 \%$ (24.6\%) more in a welfare-friendly product as compared to a regular one. In conclusion, respondents seem to care about animal welfare; however many factors may act as barriers for them to exert their ethical preferences in purchasing behavior such lack of information to the consumer before and during the purchase, low availability of products, product disassociation with the animal and cost.

Keywords: Farm animal. Demand. Availability. Consumer market. Products of animal origin.

\section{Introdução}

Nos últimos anos, no âmbito do mercado de produtos de origem animal, percebe-se uma preocupação crescente dos consumidores com a qualidade dos alimentos adquiridos. Atributos como saúde, segurança alimentar, preocupações com o meio ambiente e questões éticas são constantemente ponderados pelos consumidores no momento da compra (Napolitano et al., 2010). 0 bem-estar animal (BEA) igualmente passou a ser considerado um tema importante, sendo apontado como um assunto emergente na América Latina devido a possíveis impactos sobre a saúde animal, viabilidade econômica e percepção dos consumidores (Tadich et al., 2010).

No Brasil, o Ministério da Agricultura, Pecuária e Abastecimento (MAPA) implementou a Comissão de Bem-estar Animal, que promove discussões em torno do tema nas cadeias produtivas. Dessa forma, é possível observar esforços e iniciativas para a promoção da qualidade de vida dos animais, entretanto, ainda existem lacunas com relação a recomendações de BEA. A Comissão Europeia, em sua estratégia de BEA para o período de 20122015, utilizou a seguinte frase para simbolizar sua abordagem: "todos são responsáveis" (European Commission, 2011). Embora os envolvidos na produção animal possuam diferentes perspectivas quanto ao BEA, é possível reduzir potenciais conflitos, mostrando os benefícios que a implantação de normas de BEA poderá trazer à sociedade, aos animais e aos profissionais e produtores (Dawkins, 2017). Portanto, objetivos econômicos podem ser compatíveis com melhores padrões de bem-estar na produção animal quando o consumidor estiver apto a perceber tal atributo no produto e estiver disposto a arcar com um maior custo (Verbeke, 2009). Assim, as atitudes do mercado consumidor em relação ao BEA necessitam ser avaliadas constantemente, a fim de reportar a importância do tema à sociedade. Os objetivos deste trabalho foram estudar e identificar as atitudes dos consumidores de produtos de origem animal com ênfase no bemestar animal, possíveis vantagens do consumo de alimentos oriundos de melhores práticas de bemestar e fatores que possam influenciar as decisões de compra.

\section{Materiais e métodos}

A coleta de dados foi realizada por meio de questionário contendo questões fechadas, aplicado via internet, entre os meses de setembro a novembro de 2013, totalizando 402 respondentes. O questionário foi hospedado no site Google Drive e a divulgação do link foi feita por meio de e-mails de lista pessoal e publicação em redes sociais, com solicitação de envio a outros respondentes interessados, a partir dos contatos iniciais, caracterizando uma amostragem por conveniência com efeito bola de neve. 0 questionário resultou da adaptação de modelos aplicados por pesquisadores da Comunidade Europeia (Eurobarometer, 2007a; 2007b). O Eurobarometer é uma ferramenta utilizada para monitorar a opinião pública voltada à população dos países pertencentes 
à Comunidade Europeia. Tais questionários visaram coletar informações quanto à opinião pública e comportamento dos consumidores na área de bem-estar de animais de produção, focando em temas como a importância do assunto, conhecimento, percepções de padrões, legislação e comportamento de compra de consumidores de produtos de origem animal. 0 questionário foi composto de 30 questões fechadas, com respostas em opção de múltipla escolha, seguindo o padrão do Eurobarometer.

A primeira parte do questionário foi constituída de perguntas a respeito de informações gerais do consumidor, caracterizando aspectos sociodemográficos. A segunda parte baseou-se em questões relativas ao bem-estar de animais de produção. Os consumidores foram convidados a responder questões gerais de BEA, a fim de avaliar o grau de conhecimento sobre o tema. Posteriormente, as questões foram voltadas à percepção do grau de bem-estar de animais entre os sistemas produtivos, a situação do bem-estar de animais de produção no Brasil e perspectivas de melhoria. Em seguida, os consumidores foram convidados a responder questões que demonstrassem suas atitudes frente ao comportamento de compra.

\section{Análise estatística}

Os dados foram analisados de forma descritiva, utilizando o programa Excel 2010. Em casos de comparação de cenários foi utilizado o teste Kruskal-Wallis, com 5\% de significância.

\section{Resultados}

\section{Questões sociodemográficas}

As parcelas com maior expressão foram representadas pelo gênero feminino (54,2\%), com ensino superior $(68,2 \%)$ e da classe social $C$ $(42,8 \%)$ (Tabela 1). A maioria dos respondentes residia na região Sul $(69,4 \%)$, seguida pelo Sudeste $(17,2 \%)$, Centro Oeste $(7,9 \%)$, Nordeste $(2,7 \%)$ e Norte $(2,7 \%)$.
Tabela 1 - Dados demográficos dos respondentes

\begin{tabular}{llc}
\hline Variável & Subdivisões & $\begin{array}{c}\text { Percentual } \\
\text { (N = 402) }\end{array}$ \\
\hline Gênero & Feminino & $54,2 \%$ \\
& Masculino & $45,8 \%$ \\
Escolaridade & Ensino Fundamental & $1,5 \%$ \\
& Ensino Médio & $30,3 \%$ \\
& Ensino Superior & $68,2 \%$ \\
Renda & Até R\$ 1.355,99 & $8,2 \%$ \\
familiar & (Classe E) & \\
& De $R \$ 1.356,00$ a 2.711,99 & $16,4 \%$ \\
& (Classe D) & \\
& De $R \$ 2.712,00$ a 6.799,99 & $42,8 \%$ \\
& (Classe C) & \\
& De $R \$ 6.800,00$ a 13.555,99 & $23,1 \%$ \\
& (Classe B) & \\
& Acima de $R \$ 13.560,00$ & $9,5 \%$ \\
& (Classe A) & \\
\hline
\end{tabular}

\section{Bem-estar de animais de produção}

Com relação ao conhecimento sobre animais de produção, a maioria dos respondentes já visitou uma fazenda de criação de animais pelo menos uma vez $(67,7 \%)$ e uma porção significativa $(89,9 \%)$ acredita possuir algum nível de conhecimento sobre as condições na qual os animais são criados. Dentre os respondentes, $79,1 \%$ revelaram que gostariam de estar mais informados quanto à situação em que os animais são criados, e a internet seria o meio mais utilizado para acessar informações sobre o tema $(35,8 \%)$. Foi observado que $63,2 \%$ dos respondentes já ouviu falar sobre bem-estar dos animais de produção e que 93,6\% consideram o bem-estar importante em uma escala de 6 a 10 (Tabela 2).

A maioria dos respondentes que considera o BEA importante é do gênero feminino $(61,3 \%)$. A importância atribuída ao bem-estar também foi relacionada à classe social dos respondentes. Os respondentes que mais consideraram o BEA como importante pertenciam a classe $C(43,1 \%)$ e os que consideram menos importante pertenciam a classe D (37,6\%) (Figura 1). 
Tabela 2 - Respostas referentes a questões gerais de bem-estar animal

\begin{tabular}{|c|c|}
\hline Questões/Respostas & $\begin{array}{l}\text { Percentual } \\
(\mathrm{N}=402)\end{array}$ \\
\hline \multicolumn{2}{|l|}{$\begin{array}{l}\text { Você já visitou alguma fazenda de criação de } \\
\text { animais? }\end{array}$} \\
\hline Não, nunca & $32,3 \%$ \\
\hline Sim, uma vez & $19,2 \%$ \\
\hline Sim, de duas a três vezes & $10,9 \%$ \\
\hline Sim, mais de três vezes & $37,6 \%$ \\
\hline \multicolumn{2}{|c|}{$\begin{array}{l}0 \text { quanto acha que conhece sobre as condições em } \\
\text { que os animais são criados no Brasil? }\end{array}$} \\
\hline Nada & $10,2 \%$ \\
\hline Um pouco & $60,9 \%$ \\
\hline Muito & $28,9 \%$ \\
\hline \multicolumn{2}{|c|}{$\begin{array}{l}\text { Você gostaria de estar mais informado em relação a } \\
\text { como os animais são criados no Brasil? }\end{array}$} \\
\hline $\operatorname{Sim}$ & $79,1 \%$ \\
\hline Talvez & $15,9 \%$ \\
\hline Não & $5,0 \%$ \\
\hline \multicolumn{2}{|c|}{$\begin{array}{l}\text { Se você estivesse procurando informações sobre como } \\
\text { os animais são criados em seu país, qual (is) } \\
\text { das seguintes fontes usaria? }\end{array}$} \\
\hline Internet & $35,9 \%$ \\
\hline Revistas & $14,2 \%$ \\
\hline Televisão & $14,0 \%$ \\
\hline Livros ou informativos & $13,4 \%$ \\
\hline Jornais & $9,6 \%$ \\
\hline Discussão com amigos, parentes e colegas & $9,6 \%$ \\
\hline Rádio & $3,2 \%$ \\
\hline Não procuraria por tal informação & $0,1 \%$ \\
\hline \multicolumn{2}{|c|}{$\begin{array}{l}\text { Você já ouviu falar sobre o bem-estar dos animais de } \\
\text { produção? }\end{array}$} \\
\hline Sim & $63,2 \%$ \\
\hline Não & $36,8 \%$ \\
\hline \multicolumn{2}{|c|}{ Diga em uma escala de 1 a 10, quão importante é } \\
\hline \multicolumn{2}{|c|}{ para você o bem-estar dos animais de produção? } \\
\hline 1 a 4 & $3,9 \%$ \\
\hline 5 & $2,5 \%$ \\
\hline 6 a 10 & $93,6 \%$ \\
\hline
\end{tabular}

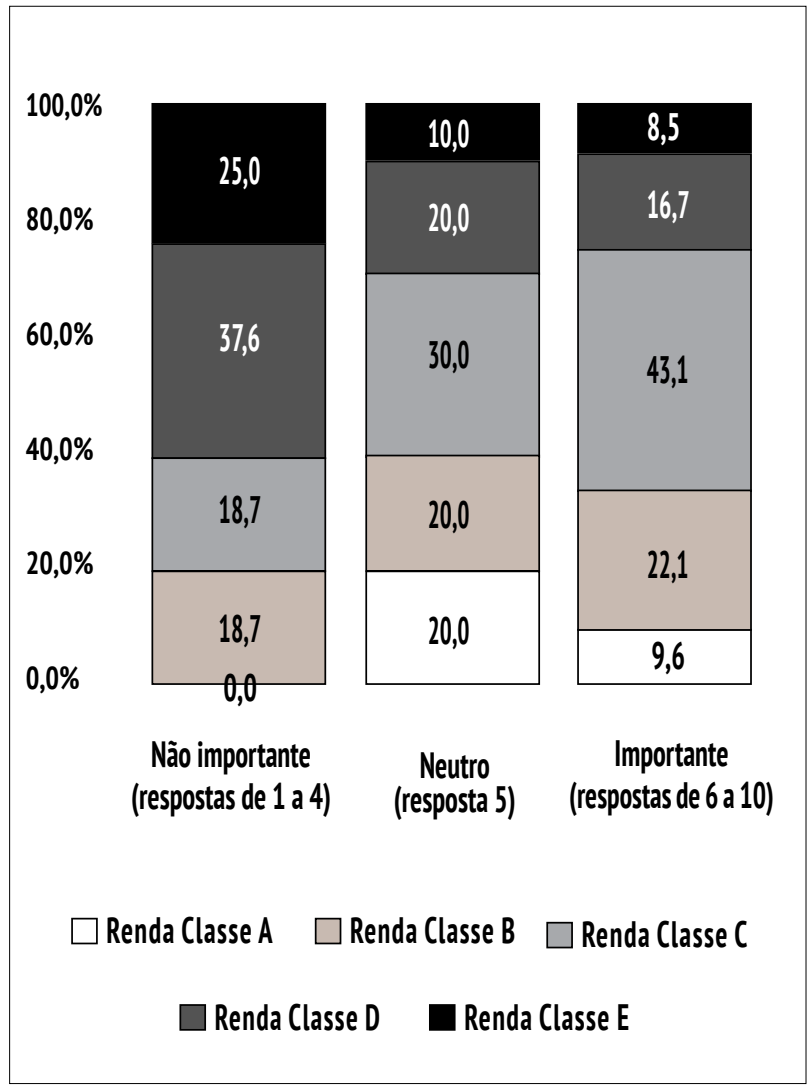

Figura 1 - Classificação das respostas quanto à importância atribuída ao bem-estar animal.

\section{Percepção sobre o bem-estar de animais de produção}

A maioria dos respondentes $(60,2 \%)$ apresentou uma visão mais negativa sobre o bem-estar de frangos de corte, suínos e galinhas poedeiras quando comparado com o bem-estar de gado de leite e de corte ( $\mathrm{P}<0,05$; Figura 2$)$.

Quanto às percepções de bem-estar dos animais de produção no Brasil, $84,6 \%$ acreditaram que melhorias ainda são necessárias, mas houve uma evolução do bem-estar entre os anos de 2003 a 2013 (69,3\%). Os produtores são considerados os maiores responsáveis em proporcionar bem-estar para os animais de produção $(43,8 \%)$. A maioria dos respondentes afirmou que a política de agricultura do Brasil revela pouco interesse pelo tema bem-estar $(82,3 \%)$ e que os animais de nosso país apresentam baixo grau de bem-estar quando comparados com os de outros países $(48,0 \%)$ (Tabela 3). 
Tabela 3 - Respostas referentes ao bem-estar dos animais de produção

\begin{tabular}{|c|c|}
\hline Questões/Respostas & $\begin{array}{l}\text { Percentual } \\
(\mathrm{N}=402)\end{array}$ \\
\hline \multicolumn{2}{|c|}{$\begin{array}{l}\text { Você acha que, em geral, o bem-estar dos animais de } \\
\text { produção no Brasil precisa ser melhorado? }\end{array}$} \\
\hline Sim & $84,6 \%$ \\
\hline Talvez & $15,4 \%$ \\
\hline Não & $0,0 \%$ \\
\hline \multicolumn{2}{|c|}{$\begin{array}{l}\text { Em geral, nos últimos } 10 \text { anos, você acha que o } \\
\text { bem-estar dos animais de produção: }\end{array}$} \\
\hline Melhorou & $63,9 \%$ \\
\hline Permaneceu o mesmo & $13,7 \%$ \\
\hline Piorou & $5,2 \%$ \\
\hline Não sei & $17,2 \%$ \\
\hline \multicolumn{2}{|c|}{$\begin{array}{l}\text { Quem você acha que deveria ser o principal } \\
\text { responsável por garantir que os animais sejam } \\
\text { produzidos com um grau aceitável de bem-estar? }\end{array}$} \\
\hline Produtores que criam os animais & $43,8 \%$ \\
\hline Governo & $21,4 \%$ \\
\hline Agroindústrias & $12,2 \%$ \\
\hline Consumidores & $10,9 \%$ \\
\hline Veterinários & $10,0 \%$ \\
\hline Mercados e restaurantes & $1,7 \%$ \\
\hline \multicolumn{2}{|c|}{$\begin{array}{l}\text { Em relação à política atual de agricultura no Brasil, } \\
\text { você acredita que o BEA recebe: }\end{array}$} \\
\hline Muita importância & $2,8 \%$ \\
\hline Nível adequado de importância & $14,9 \%$ \\
\hline Pouca importância & $82,3 \%$ \\
\hline \multicolumn{2}{|c|}{$\begin{array}{l}\text { Você acredita que o bem-estar dos animais } \\
\text { produzidos no Brasil é: }\end{array}$} \\
\hline Melhor que outros países & $11,5 \%$ \\
\hline Igual a outros países & $40,5 \%$ \\
\hline Pior que outros países & $48,0 \%$ \\
\hline
\end{tabular}

\section{Comportamento de compra}

A maioria dos consumidores entrevistados afirmou adquirir carne, leite e derivados uma vez por semana $(32,1 \%$ e $36,8 \%$, respectivamente) (Figura 3).

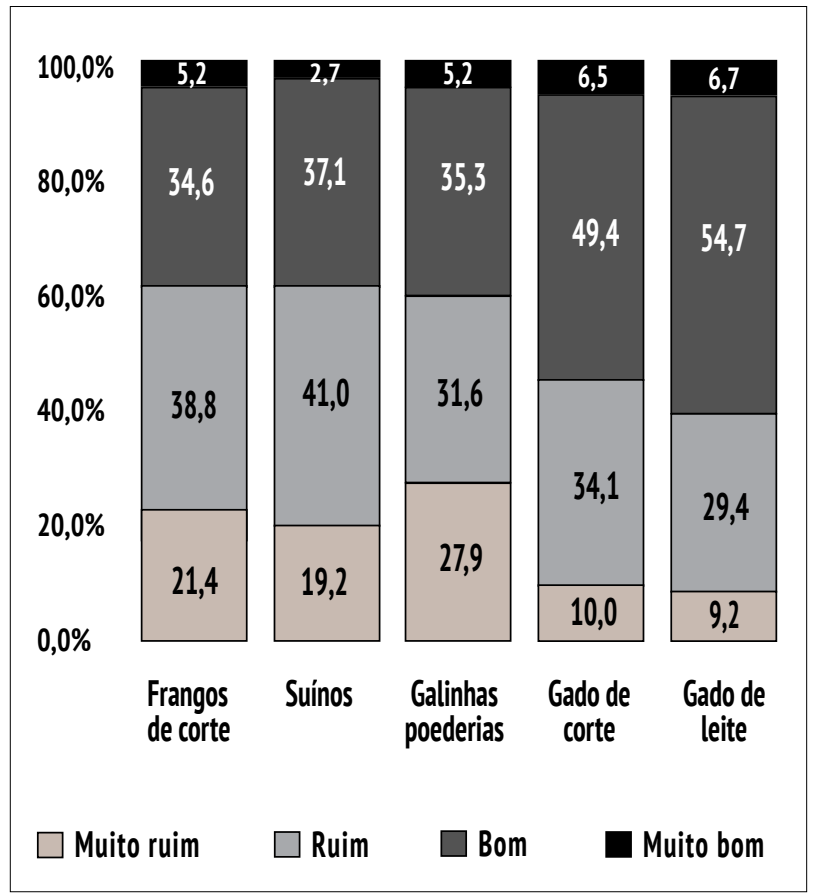

Figura 2 - Classificação dos respondentes quanto ao grau de bem-estar animal de diferentes cadeias produtivas.

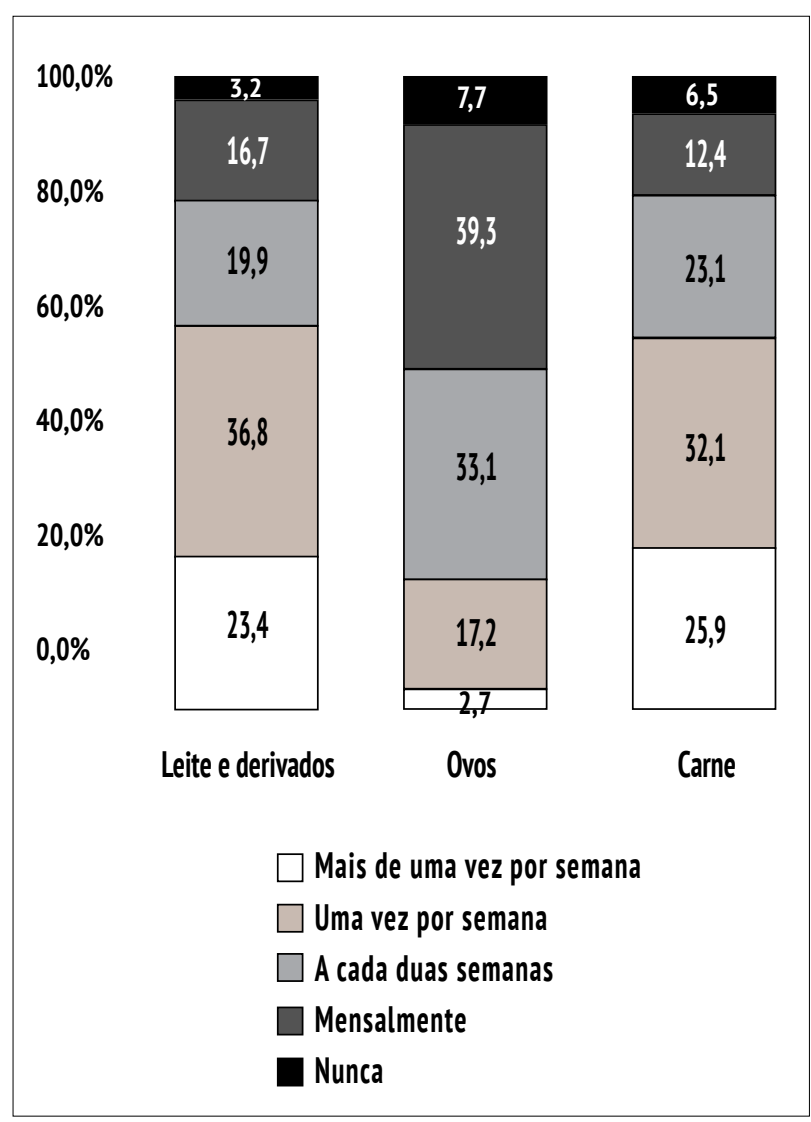

Figura 3 - Frequência de compra de produtos de origem animal. 
Quanto à importância do BEA na decisão de compra, grande parte dos respondentes (54\%) afirmou não pensar sobre o assunto no momento em que adquire produtos de origem animal. Somente $28,9 \%$ afirmam ter adquirido produtos de origem animal com maior grau de bem-estar, sendo os ovos os mais consumidos (56\%). A pesquisa ainda revelou que $88,3 \%$ dos respondentes acredita que o fato de adquirirem produtos com maior grau de bem-estar pode trazer um impacto direto na forma na qual os animais são criados (Tabela 4).

Tabela 4 - Informações gerais de bem-estar animal e comportamento de compra dos respondentes

\begin{tabular}{|c|c|}
\hline Questões/Respostas & $\begin{array}{l}\text { Porcentual } \\
(\mathrm{N}=402)\end{array}$ \\
\hline \multicolumn{2}{|c|}{$\begin{array}{l}\text { Quando você compra carne/leite/ovos, você pensa } \\
\text { sobre o bem-estar dos animais envolvidos na } \\
\text { produção do alimento em questão? }\end{array}$} \\
\hline Sim, na maioria das vezes & $13,4 \%$ \\
\hline Sim, algumas vezes & $17,2 \%$ \\
\hline Não, raramente & $54,0 \%$ \\
\hline Não, eu nunca penso nisso & $15,2 \%$ \\
\hline Eu nunca compro tais produtos & $0,2 \%$ \\
\hline \multicolumn{2}{|c|}{$\begin{array}{l}\text { Você já adquiriu um produto de origem animal } \\
\text { produzido com maior grau de bem-estar? }\end{array}$} \\
\hline Sim & $28,9 \%$ \\
\hline Não & $60,9 \%$ \\
\hline Não sei & $10,2 \%$ \\
\hline \multicolumn{2}{|c|}{ Caso já tenha adquirido tais produtos, quais? } \\
\hline Ovos & $56,0 \%$ \\
\hline Carne de frango & $24,1 \%$ \\
\hline Carne bovina & $13,9 \%$ \\
\hline Leite & $6,0 \%$ \\
\hline Suínos & $0,0 \%$ \\
\hline $\begin{array}{l}\text { Você acredita que o fato dos co } \\
\text { adquirirem produtos com alto } \mathrm{g} \\
\text { bem-estar pode trazer um impa } \\
\text { no bem-estar dos animais? }\end{array}$ & \\
\hline Com certeza & $56,0 \%$ \\
\hline Sim & $32,3 \%$ \\
\hline Talvez & $8,5 \%$ \\
\hline Não & $3,2 \%$ \\
\hline
\end{tabular}

A maioria dos consumidores $(63,4 \%)$ afirmou não conseguir identificar pela embalagem quais produtos foram oriundos de um sistema no qual os animais foram criados em melhores condições de bem-estar, e 87,3\% acreditam que, em mercados, os consumidores não conseguem encontrar facilmente informações sobre a origem e tipo de sistema em que os animais foram criados. Dentre os respondentes, $54 \%$ elegeram as etiquetas como a melhor forma de identificar as condições nas quais os animais são criados, entretanto, com escolhas insuficientes de produtos com maior grau de bem-estar $(35,6 \%)$. Observou-se que $47 \%$ mostraram-se dispostos a alterar o local de compra, desde que pudessem ter acesso a produtos com maior grau de bem-estar, pagando até $10 \%$ a mais por estes itens $(32,3 \%)$ (Tabela 5).

Tabela 5 - Comportamento de compra dos respondentes

\begin{tabular}{|c|c|}
\hline Questões/Respostas & $\begin{array}{c}\text { Porcentual } \\
(\mathrm{N}=402)\end{array}$ \\
\hline \multicolumn{2}{|l|}{$\begin{array}{l}\text { Ao adquirir ovos, carne ou leite, você consegue } \\
\text { identificar pela etiqueta quais produtos possuem um } \\
\text { sistema em que os animais foram criados com maior } \\
\text { grau de bem-estar? }\end{array}$} \\
\hline Sempre & $1,0 \%$ \\
\hline Na maioria das vezes & $6,0 \%$ \\
\hline Algumas vezes & $29,6 \%$ \\
\hline Nunca & $63,4 \%$ \\
\hline \multicolumn{2}{|l|}{$\begin{array}{l}\text { Você acredita que, em mercados, os consumidores } \\
\text { conseguem encontrar facilmente a informação nos } \\
\text { produtos sobre a origem e o tipo de sistema em que os } \\
\text { animais são criados? }\end{array}$} \\
\hline Sim & $2,7 \%$ \\
\hline Não & $87,3 \%$ \\
\hline Não sei & $10,0 \%$ \\
\hline \multicolumn{2}{|l|}{$\begin{array}{l}\text { Qual dos itens abaixo você acredita ser a melhor } \\
\text { forma de identificar as condições em que os animais } \\
\text { foram criados? }\end{array}$} \\
\hline $\begin{array}{l}\text { Etiquetas nos produtos com informações do sistema } \\
\text { descritas }\end{array}$ & $54,0 \%$ \\
\hline $\begin{array}{l}\text { Usar um sistema de escores (por exemplo: } 5 \text { estrelas } \\
\text { para o melhor produto e } 1 \text { para o básico) }\end{array}$ & $18,4 \%$ \\
\hline Logos nos produtos & $10,4 \%$ \\
\hline
\end{tabular}


Tabela 5 - Comportamento de compra dos respondentes

(continuação)

\begin{tabular}{|c|c|}
\hline Questões/Respostas & $\begin{array}{c}\text { Porcentual } \\
(\mathrm{N}=402)\end{array}$ \\
\hline $\begin{array}{l}\text { Qual dos itens abaixo você acredita ser a melhor } \\
\text { forma de identificar as condições em que os animais } \\
\text { foram criados? }\end{array}$ & \\
\hline $\begin{array}{l}\text { Fornecer uma imagem do tipo de criação em que os } \\
\text { animais se encontravam }\end{array}$ & $5,7 \%$ \\
\hline Coloração de etiquetas & $5,0 \%$ \\
\hline Pôsteres informativos nos mercados & $4,7 \%$ \\
\hline Selos de certificação & $1,0 \%$ \\
\hline Divulgação pela internet & $0,8 \%$ \\
\hline $\begin{array}{l}\text { Você acredita que atualmente existem possibilidades } \\
\text { de escolha suficientes de produtos produzidos com } \\
\text { maior grau de bem-estar em mercados? }\end{array}$ & \\
\hline Certamente sim & $3,2 \%$ \\
\hline Provavelmente sim & $10,4 \%$ \\
\hline Não sei & $11,7 \%$ \\
\hline Provavelmente não & $39,1 \%$ \\
\hline Certamente não & $35,6 \%$ \\
\hline $\begin{array}{l}\text { Você estaria disponível a trocar o local em que } \\
\text { normalmente adquire alimentos por um local que } \\
\text { oferecesse maior variedade de produtos produzidos } \\
\text { com maior grau de bem-estar? }\end{array}$ & \\
\hline Certamente sim & $47,0 \%$ \\
\hline Provavelmente sim & $40,8 \%$ \\
\hline Não sei & $4,0 \%$ \\
\hline Provavelmente não & $7,5 \%$ \\
\hline Certamente não & $0,7 \%$ \\
\hline $\begin{array}{l}\text { Quanto você aceitaria pagar a mais por produtos com } \\
\text { maior grau de bem-estar? }\end{array}$ & \\
\hline $5 \%$ a mais & $28,4 \%$ \\
\hline $10 \%$ a mais & $32,3 \%$ \\
\hline $25 \%$ a mais & $24,6 \%$ \\
\hline Acima de $25 \%$ & $14,7 \%$ \\
\hline
\end{tabular}

\section{Discussão}

Quanto à escolaridade dos respondentes, 68,2\% tinha ensino superior completo, o que não representa a estratificação da sociedade brasileira. Segundo o Instituto Brasileiro de Geografia e Estatística (IBGE,
2016), 13,5\% dos homens e 16,9\% das mulheres possuem escolaridade em nível de ensino superior no Brasil.

A maioria dos respondentes já ouviu falar sobre bem-estar de animais de produção e demonstraram o desejo de adquirirem mais informações sobre como são criados para que possam fazer escolhas conscientes. Além disso, em comparação ao observado por Bonamigo et al. (2012), cujo estudo mostrou que $68,5 \%$ dos entrevistados afirmaram não conhecer os sistemas de produção, os resultados desta pesquisa indicaram que as pessoas estavam mais informadas quanto à forma de criação dos animais, dado que $10,2 \%$ das pessoas não sabia em que condições os animais são criados.

Uma ferramenta que contribui com a disseminação de informações e esclarecimento de diversas questões, inclusive sobre BEA, é a internet. Cardoso et al. (2017) entrevistaram passageiros em um aeroporto de Santa Catarina e verificaram que alguns respondentes gostariam de ter mais conhecimento sobre como os animais são mantidos nos sistemas de produção e que suas fontes para coleta de informações seriam a televisão e a internet. De acordo com o Comitê Gestor da Internet no Brasil (CGI), 69\% dos brasileiros utilizavam a internet diariamente em 2012 (CGI, 2012). Considerando que a maioria dos respondentes possuia acesso à internet, uma forma interessante de ampliar o conhecimento quanto ao bem-estar de animais de produção é a oferta de textos de fácil acesso via internet.

A importância atribuída ao BEA pareceu ser influenciada pelas diferentes classes sociais e pelo gênero. A maioria dos consumidores julgaram o BEA importante, porém o rendimento familiar revelou-se como uma barreira para a consideração da importância do tema. Com relação ao gênero dos respondentes, em uma revisão de 31 trabalhos que estudaram a correlação do gênero com a interação entre seres humanos e animais, observouse que mulheres apresentaram maior empatia com os animais do que homens (Herzog, 2007) e mostraram-se mais atentas às questões relacionadas ao BEA (Heise e Theuvsen, 2017).

A visão negativa dos respondentes com relação ao bem-estar de animais de produção neste estudo demonstrou ser semelhante às expressas pelos 
cidadãos europeus (European Commission, 2007). Consumidores alemães (88\%), por exemplo, consideraram que havia a necessidade de padrões mais elevados em relação ao BEA (Heise e Theuvsen, 2017). Nesse sentido, as cadeias consideradas como as que proporcionam piores condições aos animais foram as de suínos, frangos de corte e galinhas poedeiras. De maneira semelhante, Nordi et al. (2007), ao avaliarem a percepção de residentes na cidade de Curitiba/PR, observaram que a criação de suínos e frangos de corte foi citada como a causadora de maior grau de sofrimento animal dentre os sistemas de produção. Tais percepções demonstram similaridades com o diagnóstico técnico de BEA nas cadeias produtivas, pois os sistemas industriais de produção de ovos (Dawkins, 2006) e suínos (Machado Filho e Hotzel, 2000) foram considerados os sistemas com o maior grau de sofrimento animal.

Neste estudo, 48\% dos respondentes consideraram o bem-estar dos animais produzidos no Brasil como pior do que em outros países. Estudos de Y unes et al. (2017) revelaram a mesma percepção para $79 \%$ dos respondentes em um estudo sobre as crenças e atitudes de brasileiros em relação aos sistemas de produção animal, enfatizando questões éticas relacionadas à qualidade de vida dos animais e reconhecendo que os mesmos deveriam ser respeitados, com o atendimento pleno de suas necessidades básicas. Entretanto, tal percepção não corresponde com o observado por Tuyttens et al. (2015), os quais encontraram indicadores de bem-estar em frangos de corte criados no Brasil superiores aos frangos de corte criados na Bélgica em situações similares.

Mais da metade dos respondentes (64\%) acredita não existir legislação que regulamente as condições de bem-estar de animais de produção criados no país. No entanto, o regulamento técnico para abate humanitário de animais de produção, estabelecido por meio da instrução normativa número 03 (MAPA, 2000) e pelo Decreto número 9.013 de 29 de março de 2017 (RIISPOA, 2017), especifica normas de BEA em torno do abate e inspeção industrial e sanitária dos produtos de origem animal. A instrução normativa número 56 (MAPA, 2008) estabeleceu recomendações gerais de boas práticas de bem-estar para animais de produção no nascimento, criação e transporte, porém sem detalhamento de situações específicas sobre a forma como os animais são criados, como formas de manejo e alojamento. A resolução número 675 de 21 de junho de 2017 igualmente estabelece normas específicas no que tange o transporte de animais de produção, ou interesse econômico, esporte, lazer e exposição por veículo automotor, de modo a evitar sofrimento desnecessário e garantir o bem-estar das espécies em trânsito (Contran, 2017). Além disso, existem normas de proteção animal no Brasil que contemplam todos os animais, incluindo animais de produção. As legislações brasileiras consideram como crime atos de crueldade e maus-tratos contra animais (Brasil, 1988; Brasil, 1998).

Dos respondentes, $10,2 \%$ relataram não saber se já adquiriram alimentos produzidos com maior grau de bem-estar, demonstrando falha na divulgação de informação e forma de demonstração de tais produtos, o que pode causar impactos diretos no comportamento de compra. A disponibilidade dos produtos com maior grau de BEA e seus valores superiores aos demais produtos podem ser consideradas barreiras para o consumo destes itens. Segundo Franco et al. (2018), um total de 52,8\% dos varejistas entrevistados na cidade de Curitiba/PR, mantinham estoques de produtos com maior grau de BEA, sendo a maioria de ovos $(41,7 \%)$, carne de frango $(38,8 \%)$ e leite $(8,3 \%)$. Dos consumidores que já adquiriram produtos com maior grau de bemestar, $56 \%$ afirmaram ter adquirido ovos e $24,1 \%$ carne de frango, provavelmente devido à maior disponibilidade de tais produtos em mercados. 0 consumo de tais produtos também se demonstra superior em países da Europa (Eurobarometer, 2007a, 2007b).

A pesquisa revelou que a maioria dos consumidores $(88,3 \%)$ acredita que o fato de adquirirem produtos com maior grau de bemestar pode trazer um impacto na forma em que os animais são criados. Em estudo similar, 52\% dos consumidores norte-americanos demonstraram possuir tal percepção (Lusk et al., 2007). Os mesmos autores relataram que melhorias no BEA de animais de produção podem ser impulsionadas pela demanda do mercado e não somente pelo governo. Assim, a percepção de responsabilidade por um tratamento satisfatório dos animais é um fator determinante do comportamento de compra, pois 
consumidores irão adquirir produtos associados a maior grau de BEA se eles se sentirem responsáveis por garantir que animais são bem tratados e que seu comportamento de compra pode fazer uma diferença na vida dos animais (Toma et al., 2012).

Os consumidores afirmaram não identificar adequadamente produtos provenientes de sistemas com alto grau de BEA. Tais resultados se demonstraram como entrave para o consumo de produtos com maior grau de BEA, uma vez que a informação é acessada pelos consumidores apenas no momento da compra. Embora os envolvidos com a produção animal conheçam sua forma de criação, os consumidores em geral não conseguem observar ou verificar tal informação. A exemplo disso, Heise e Theuvsen (2017) observaram em seus estudos que, em geral, os consumidores alemães classificaram seus próprios conhecimentos sobre agricultura como baixo, indicando que estão mal informados sobre tais temas. Assim, na falta de uma informação confiável e em detalhamento suficiente sobre a forma com que os animais são criados, os consumidores não possuem certeza sobre tais características (Lusk et al., 2007).

0 preço demonstrou ser outro fator determinante no comportamento de compra. Neste estudo, embora a maioria das pessoas $(32,3 \%)$ estivesse disposta a pagar $10 \%$ a mais por produtos oriundos de sistemas com maior grau de BEA, 54\% dos respondentes reportaram não pensar sobre BEA no momento da compra dos alimentos. Esses resultados podem ser explicados pelo exposto por Tuyttens et al. (2014), os quais citaram que os indivíduos relatam uma grande importância ao BEA em seu papel como cidadão, entretanto, não se comportam dessa maneira no momento em que se tornam consumidores, pois não apresentam ações nas quais tenham feito investimentos para melhorar o bem-estar dos animais. Essa lacuna entre comportamento e atitude é conhecida como dualidade entre consumidor e cidadão (Vanhonacker et al., 2008). Consumidores brasileiros atribuem o preço e qualidade da carne como atributos de maior importância no momento da compra (Bonamigo et al., 2012; Franchi et al., 2012). Inicialmente, o BEA não é levado em consideração, e isso pode ser explicado parcialmente pela falta de informação do consumidor sobre os modelos de produção adotados. Viegas et al. (2015) relataram que o BEA somente é mencionado após uma pergunta direta sobre o assunto. Entretanto, quando uma reflexão sobre o BEA é estimulada, a maioria dos consumidores mostra-se disposta a pagar um valor adicional pelo produto com tal atributo (Bonamigo et al., 2012), resultado igualmente observado em pesquisas com consumidores mexicanos, dos quais $68,2 \%$ foram positivos ao fato de pagarem mais por produtos mais favoráveis aos animais, sendo aqueles residentes nas cidades os mais dispostos a pagar mais em comparação com os consumidores rurais (Miranda-de la Lama et al., 2017).

O comportamento de compra é formado por uma combinação de atributos, na qual se deixa de escolher um atributo que se considera importante em função da combinação formada por outros. Melhorias em BEA podem resultar em desvantagens como maior custo e consequente redução da aceitação do consumidor (Tuyttens et al., 2014), especialmente em situações de baixa renda familiar, quando os consumidores preferem a escolha de menor preço (Fisher e Bowles, 2002). No entanto, o aumento do custo de produção agregado a melhores práticas de BEA pode ser compensado pelo aumento do preço de venda do produto diferenciado, inserindo questões éticas que são importantes para o consumidor. Ainda que a compra dos produtos com maior BEA esteja condicionada à renda familiar, a oportunidade de escolha e acesso à informação são cruciais tanto para os consumidores quanto para o fomento de estratégias que beneficiem a qualidade de vida dos animais.

\section{Conclusão}

Os consumidores demonstraram que consideram o bem-estar de animais de produção importante e percebem sua influência ao adquirirem produtos diferenciados para tal atributo. 0 fato de os altos níveis de preocupação revelados na pesquisa não se traduzirem necessariamente em comportamento de compra não parece relacionado exclusivamente à falta de preocupação dos consumidores. 0 problemachave parece estar relacionado às diversas barreiras para a adoção de tal comportamento, como falta de informação disponível para o consumidor antes e 
no momento da compra, baixa disponibilidade dos produtos, desassociação do produto com o animal de origem e custo. Tais barreiras podem fazer com que os consumidores não exerçam sua preferência ética em escolha de compra. Neste sentido, sugerese que esforços sejam feitos no sentido de resolver as barreiras existentes para que o consumidor exerça sua escolha ética durante o comportamento de compra.

\section{Referências}

Bonamigo A, Bonamigo CBSS, Molento CFM. Atribuições da carne de frango relevantes ao consumidor: foco no bem-estar animal. R Bras Zootec. 2012;41(4):1044-50.

Brasil. Constituição da República Federativa do Brasil. Diário Oficial da República Federativa do Brasil; 1998, nº 191-A, seção I, p. 1.

Brasil. Lei no 9.605, de 12 de fevereiro de 1998. Dispõe sobre as sanções penais e administrativas derivadas de condutas e atividades lesivas ao meio ambiente, e dá outras providências. Diário Oficial da República Federativa do Brasil; 1998, seção 1, 13 fev, p. 1.

Cardoso CS, von Keyserlingk MAG, Hötzel MJ. Brazilian citizens: expectations regarding dairy cattle welfare and awareness of contentious practices. Animals (Basel). 2017;7(12):E89.

CGI - Comitê Gestor da Internet no Brasil. TIC Domicílios e Empresas - Pesquisa sobre o uso das tecnologias de informação e Comunicação no Brasil. 2012 [acesso 5 fev 2018]. Disponível em: https://tinyurl.com/yboxu594.

Contran. Conselho Nacional de Trânsito. Resolução no․ 675 de 21 de junho de 2017. Dispõe sobre o transporte de animais de produção ou interesse econômico, esporte, lazer e exposição. [acesso 24 mai 2018]. Disponível em: https://tinyurl.com/yaqt6nky.

Dawkins MS. A user's guide to animal welfare science. Trends Ecol Evol. 2006;21(2):77-82.

Dawkins MS. Animal welfare and efficient farming: is conflict inevitable? Anim Prod Sci. 2017;57:201-8.
Eurobarometer. Attitudes of EU citizens towards Animal Welfare. Special Eurobarometer 270/Wave 66.1 - TNS Opinion \& Social. 2007a [acesso 24 mai 2018]. Disponível em: https://tinyurl.com/y9w9wk49.

Eurobarometer. Attitudes of consumers towards the welfare of farmed animals - Wave 2. 2007b [acesso 24 mai 2018]. Disponível em: https://tinyurl.com/y7txuele.

European Commission. Communication from the Commission to the European Parliament, the Council, the European Economic and Social Committee and the Committee of the Regions. A renewed EU strategy for 2011-2014 for Corporate Social Responsibility. Bruxelas; 2011 [acesso 24 mai 2018]. Disponível em: https:// tinyurl.com/k7dky5t.

European Commission. EU consumers willing to pay for better animal welfare. Bruxelas; 2007 [acesso 10 jan 2018]. Disponível em: https://tinyurl.com/y8khhznc.

Fisher C, Bowles D. Hard-boiled reality: animal welfarefriendly egg production in a global market. Horsham, UK: Royal Society for the Prevention of Cruelty to Animals (RSPCA); 2002.

Franco BMR, Souza APO, Molento CFM. Welfare-friendly products: availability, labeling and opinion of retailers in Curitiba, Southern Brazil. Rev Econ Sociol Rural. 2018; 56(1):9-18.

Franchi GA, Nunes MLA, Garcia PR, Silva IJO. Percepção do mercado consumidor de Piracicaba em relação ao bem-estar dos animais de produção. PUBVET. 2012;6(11):1325-30.

Heise H, Theuvsen L. What do consumers think about farm animal welfare in modern agriculture? Attitudes and shopping behaviour. Int Food Agribus Man. 2017;20(3):379-99.

Herzog HA. Gender differences in human-animal interactions: A review. Anthrozoos. 2007;20(1):7-21.

IBGE - Instituto Brasileiro de Geografia e Estatística. Pesquisa Nacional por Amostra de Domicílios Contínua, 2016 [acesso 20 fev 2018]. Disponível em: https:// tinyurl.com/y9apfpr4. 
Lusk JL, Nilsson T, Foster K. Public preferences and private choices: effect of altruism and free riding on demand for environmentally certified pork. Environ Resour Econ. 2007;36(4):499-521.

Miranda-de la Lama GC, Estévez-Moreno LX, Sepúlveda WS, Estrada-Chavero MC, Rayas-Amor AA, Villarroel M, et al. Mexican consumers' perceptions and attitudes towards farm animal welfare and willingness to pay for welfare friendly meat products. Meat Sci. 2017;125:106-13.

MAPA - Ministério da Agricultura, Pecuária e Abastecimento. Instrução normativa $\mathrm{n}^{\circ} 3$, de 17 de janeiro de 2000. Regulamento técnico de métodos de insensibilização para o abate humanitário de animais de açougue. [acesso 24 mai 2018]. Disponível em: https:// tinyurl.com/yb8d6a3w.

MAPA-MinistériodaAgricultura,PecuáriaeAbastecimento. Instrução Normativa no 56 de 6 de novembro de 2008. Estabelece os procedimentos gerais de Recomendações de Boas Práticas de Bem-Estar para Animais de Produção e de Interesse Econômico - REBEM, abrangendo os sistemas de produção e transporte. [acesso 24 mai 2018]. Disponível em: https://tinyurl.com/y7mhxyc8.

Napolitano F, Girolami A, Braghieri A. Consumer liking and willingness to pay for high welfare animal-based products. Trends Food Sci Technol. 2010;21(11):537-43.

Nordi WM, Bones VC, Stupack EC, Soares DR, Molento CFM. Percepção e atitude em relação ao bem-estar de animais de produção em Curitiba, Paraná. XVII Congresso Brasileiro de Zootecnia - ZOOTEC; 20 mai - 1 jun 2007; Londrina, Paraná. Londrina: ABZ; 2007.

Machado Filho LCP, Hötzel MJ. Bem-estar dos suínos. 5 Seminário Internacional de Suinocultura; 27-28 set 2000; São Paulo. Concórdia: Embrapa Suínos e Aves; 2000. p. 70-82.
RIISPOA - Regulamento e Inspeção Industrial e Sanitária de produtos de Origem Animal. Decreto no 9.013 de 29 de março de 2017. Regulamenta as Leis no 1.283 e no 7.889 que dispõem sobre a inspeção industrial e sanitária de produtos de origem animal. [acesso 24 mai 2018]. Disponível em: https://tinyurl.com/yb55xnat.

Tadich NA, Molento CFM, Gallo CB. Teaching animal welfare in some veterinary schools in Latin America. J Vet Med Educ. 2010;37(1):69-73.

Toma L, Stott AW, Revoredo-Giha C, Kupiec-Teahan B. Consumers and animal welfare: comparison between European Union countries. Appetite. 2012;58(2):597-607.

Tuyttens FA, Vanhonacker F, Verbeke W. Broiler production in Flanders, Belgium: current situation and producers' opinions about animal welfare. Worlds Poult Sci J. 2014;70:343-54.

Tuyttens FA, Federici JF, Vanderhasselt RF, Goethals K, Duchateau L, Sans ECO, et al. Assessment of welfare of Brazilian and Belgian broiler flocks using the Welfare Quality protocol. Poult Sci. 2015;94(8):1758-66.

Vanhonacker F, Verbeke W, Van Poucke E, Tuyttens FAM. Do citizens and farmers interpret the concept of farm animal welfare differently? Livest Sci. 2008;116(1-3):126-36.

Verbeke W. Stakeholder, citizen and consumer interests in farm animal welfare. Anim Welf. 2009;18(4):325-33.

Viegas I, Santos JML, Fontes MA. Percepção dos consumidores relativamente à carne de bovino: cenários de escolha a partir de grupos de discussão. Rev Econ Sociol Rural. 2015;53(Supl 1):S49-62.

Yunes MC, von Keyserlingk MAG, Hötzel MJ. Brazilian citizens's opinions and attitudes about farm animal production systems. Animals (Basel). 2017;7(10):75. 